\title{
Broadband Single-Nanowire Photoconductive Terahertz Detectors
}

\author{
Kun Peng ${ }^{1}$, Patrick Parkinson², Qian Gao ${ }^{1}$, Jessica L. Boland ${ }^{3}$, Ziyuan $\mathrm{Li}^{1}$, Fan Wang ${ }^{4}$, Yesaya C. Wenas ${ }^{1}$, \\ Christopher L. Davies ${ }^{3}$, Lan Fu' ${ }^{*}$, Michael B. Johnston ${ }^{3}$, Hark Hoe Tan ${ }^{1}$, Chennupati Jagadish ${ }^{1}$ \\ 1. Department of Electronic Materials Engineering, Research School of Physics and Engineering, \\ The Australian National University, Canberra, ACT 2601, Australia \\ 2. School of Physics and Astronomy and the Photon Science Institute, University of Manchester, Manchester, M13 9PL, UK. \\ 3. Department of Physics, University of Oxford, Clarendon Laboratory, Oxford, OX1 3PU, UK. \\ 4. ARC Centre of Excellence for Nanoscale BioPhotonics (CNBP), Faculty of Science, Macquarie University, Sydney, NSW 2109, Australia. \\ *Corresponding author: Lan Fu, e-mail: lan.fu@anu.edu.au
}

\begin{abstract}
Broadband photoconductive terahertz detectors based on undoped InP single nanowires were demonstrated. By further design and growth of an axial $\mathrm{n}^{+}-\mathrm{i}-\mathrm{n}^{+}$structure to reduce the contact resistance, highly-sensitive $\mathrm{n}^{+}-\mathrm{i}-\mathrm{n}^{+} \mathrm{InP}$ single-nanowire terahertz detectors were achieved.
\end{abstract}

OCIS codes: (040.5160) Photodetectors; (160.4236) Nanomaterials; (300.6495) Spectroscopy, terahertz

\section{Introduction}

Terahertz (THz) electronics based on III-V semiconductor nanowires have been recently demonstrated as promising nano-components for advanced THz spectroscopy and imaging systems with sub-wavelength spatial resolution and broadband spectral sensitivity. We have previously demonstrated GaAs/AlGaAs single-nanowire photoconductive $\mathrm{THz}$ detectors with good performance (i.e., high signal-to-noise ratio) but only a narrow detection bandwidth [1]. In this work, by optimizing both the detector geometry and the detection material, broadband photoconductive $\mathrm{THz}$ detectors based on single InP nanowires were realized with excellent performance approaching to that of traditional bulk THz photoconductive receivers. It is also noted that Schottky contacts were formed between the nanowire and contact metal for these InP nanowire detectors, which needs to be further improved for maximum detection sensitivity. An axial $n^{+}-i-n^{+}$InP nanowire structure was then designed and investigated, leading to improved contact quality (due to contact doping) and thus further improvement of the InP single-nanowire detector, particularly in its signal-to-noise ratio.

\section{Nanowire growth and detector fabrication}

The InP nanowires studied in this work were grown by selective-area metal organic vapor phase epitaxy technique [2], as shown in Fig. 1(a) and (b). For $\mathrm{n}^{+}$doping, gaseous silane was introduced during nanowire growth to produce Si-doped, n-type nanowire segments. All nanowires have a wurtzite structure and are free of stacking faults. A novel, non-contact, microscopic technique [3] combining both power-dependent photoluminescence (PL) and timeresolved PL measurements were employed to profile the doping concentration along the single nanowire for design and characterization of $\mathrm{n}^{+}-\mathrm{i}-\mathrm{n}^{+}$nanowire structure. The resultant doping profiles of an undoped single InP nanowire and an $\mathrm{n}^{+}-\mathrm{i}-\mathrm{n}^{+} \mathrm{InP}$ single nanowire studied in this work are shown in Fig. 1(c) and (d), respectively.
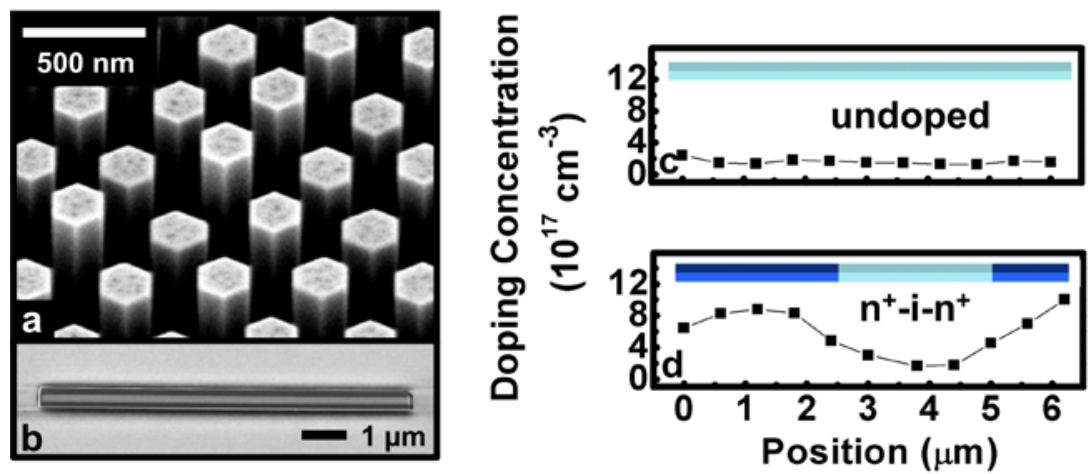

Fig. 1. SEM images of the InP nanowires used in this study: a) an array of nanowires; b) a single nanowire transferred onto a z-cut quartz substrate. Doping profiles of single InP nanowires with c) unintentional and d) $n^{+}-i-n^{+}$doping as determined by our new optical technique. 
To fabricate the single-nanowire THz detectors, conventional UV photolithography was employed to pattern the electrodes onto the nanowires. A strip-line antenna structure was chosen, as this has previously been proven to provide a broadband THz response in bulk detectors [4]. An oxygen plasma etch was used for further removal of the photoresist residue on nanowires after patterning, followed by a $9.3 \% \mathrm{HCl}$ chemical etching to remove native oxide layer formed on the nanowire surface. Finally, the detector structures were metallized using electron beam evaporation, followed by lift-off to form Ti/Au $(10 \mathrm{~nm} / 300 \mathrm{~nm})$ contacts, as shown in Fig. 2(a).
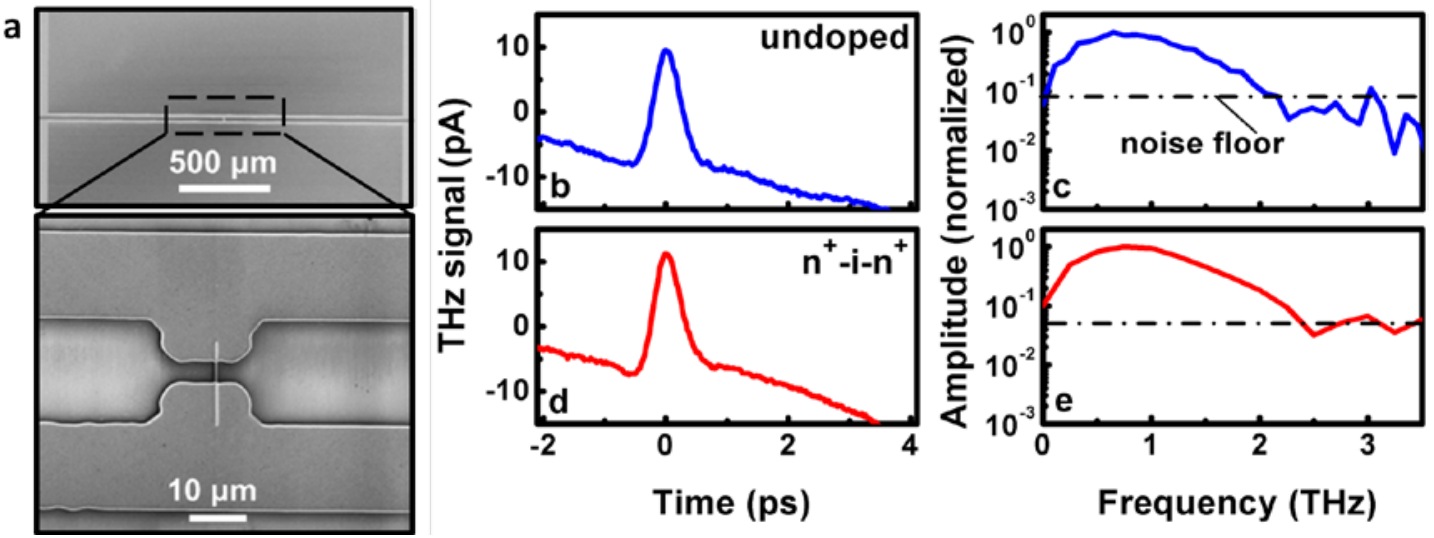

Fig.2. a) SEM images of the InP single-nanowire THz detector used in this work. b) and d) unprocessed time-domain THz response current, c) and e) $\mathrm{THz}$ response spectra measured from an undoped (blue) and an $\mathrm{n}^{+}-\mathrm{i}-\mathrm{n}^{+}$(red) InP single-nanowire detector in a THz-TDS system.

\section{THz response characterization}

The undoped and $\mathrm{n}^{+}-\mathrm{i}-\mathrm{n}^{+} \mathrm{InP}$ single-nanowire detectors were incorporated into a pulsed $\mathrm{THz}$ time-domain spectroscopy (THz-TDS) system [1,4] for characterization as THz detectors. Fig. 2(b)-(e) show typical THz

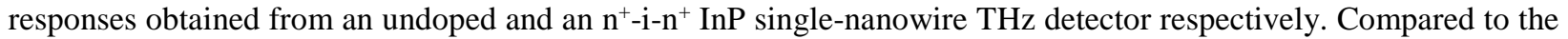
undoped nanowire detectors, a significant performance enhancement including an improved detection bandwidth as well as 2.5-time increased signal-to-noise ratio was observed for the $\mathrm{n}^{+}-\mathrm{i}-\mathrm{n}^{+}$nanowire $\mathrm{THz}$ detector compared to the undoped nanowire detectors, which is as good as those of a traditional bulk $\mathrm{InP} \mathrm{THz}$ detector despite that the nanowire detectors have only pA-level response current due to the nano-size active material volume.

\section{Conclusion}

This work confirms the suitability of III-V nanowires detectors in THz-TDS applications, bringing the promise of nanowire-based THz electronics closer to industrial applicability. Moreover, the doping engineering introduced in this work is also highly applicable for design and development of efficient single-nanowire based photoconductive emitters.

\section{References}

[1] K. Peng, P. Parkinson, L. Fu, Q. Gao, N. Jiang, Y-N Guo, F Wang, H. J. Joyce, J. L. Boland, H. H. Tan, C. Jagadish and M. B. Johnston, "Single nanowire photoconductive terahertz detectors," NANO LETT 15, 206-210,(2015).

[2] Q. GaO, D. Saxena, F. Wang, L. Fu, S. Mokkapati, Y. A. Guo, L. Li, J. Wong-Leung, P. Caroff, H. H. Tan, and C. Jagadish, "Selective-Area Epitaxy of Pure Wurtzite InP Nanowires: High Quantum Efficiency and Room-Temperature Lasing," NANO LETT 14, 5206-5211, (2014)

[3] F. Wang, Q. Gao, K. Peng, Z. Li, Z. Y. Li, Y. N. Guo, L. Fu, L. M. Smith, H. H. Tan, and C. Jagadish, "Spatially Resolved Doping Concentration and Nonradiative Lifetime Profiles in Single Si-Doped InP Nanowires Using Photoluminescence Mapping," NANO LETT 15, 153017-3023, (2015).

[4] K. Peng, P. Parkinson, J. L. Boland, Q. Gao, Y. C. Wenas, C. L. Davies, Z. Li, L. Fu, M. B. Johnston, H. H. Tan, and C. Jagadish, "Broadband Phase-Sensitive Single InP Nanowire Photoconductive Terahertz Detectors," NANO LETT 16, 4925-4931, (2016) 